\title{
Efeitos hemogasométricos da xilazina e da romifidina em cabras tratadas por ioimbina
}

\author{
[Haemogasometric effects of xylazine and romifidine in goats treated with yohimbine] \\ J.A.B. Bastos ${ }^{1}$, F.O. Paes Leme $^{2}$, G.E.S. Alves ${ }^{3 *}$ \\ ${ }^{1}$ Médico Veterinário - MS \\ ${ }^{2}$ Faculdade de Jaguariúna - Jaguariúna, SP \\ ${ }^{3}$ Escola de Veterinária da Universidade Federal de Minas Gerais \\ Caixa Postal 567 \\ 30123-970 - Belo Horizonte, MG
}

\begin{abstract}
RESUMO
Estudaram-se as alterações produzidas por doses equipotentes de xilazina e romifidina e os efeitos da administração subseqüente de ioimbina em oito cabras mestiças. Respeitou-se um intervalo de sete dias entre os seguintes tratamentos: A- $250 \mu \mathrm{g} / \mathrm{kg} / \mathrm{IM}$ de xilazina e $0,1 \mathrm{ml} / \mathrm{kg} / \mathrm{IV}$ de solução fisiológica, B$250 \mu \mathrm{g} / \mathrm{kg} / \mathrm{IM}$ de xilazina e $250 \mu \mathrm{g} / \mathrm{kg} / \mathrm{IV}$ de ioimbina, C- $25 \mu \mathrm{g} / \mathrm{kg} / \mathrm{IM}$ de romifidina e $0,1 \mathrm{ml} / \mathrm{kg} / \mathrm{IV}$ de solução fisiológica, D- $25 \mu \mathrm{g} / \mathrm{kg} / \mathrm{IM}$ de romifidina e $250 \mu \mathrm{g} / \mathrm{kg} / \mathrm{IV}$ de ioimbina. Foram mensurados a freqüência respiratória, o $\mathrm{pH}$, as pressões parciais de oxigênio e dióxido de carbono, a concentração de ín bicarbonato, o excesso de bases e a saturação de oxigênio no sangue arterial. Utilizou-se um delineamento experimental crossover, e as médias foram comparadas pelo teste Duncan $(\mathrm{P} \leq 0,05)$. Xilazina e romifidina reduziram a pressão parcial de oxigênio e aumentaram a pressão parcial de dióxido de carbono. A ioimbina reverteu os efeitos da xilazina e da romifidina sobre as pressões parciais de oxigênio e dióxido de carbono no sangue arterial.
\end{abstract}

Palavras-chave: cabra, xilazina, romifidina, ioimbina

\begin{abstract}
With the purpose to assess some of the clinical and laboratorial features induced by xylazine and romifidine, at equipotent sedative doses, followed by the use of yohimbine, eight crossbred female goats were assigned randomly to four treatments at the following dose rates: A- $250 \mu \mathrm{g} / \mathrm{kg} / \mathrm{IM}$ xylazine plus $0.1 \mathrm{ml} / \mathrm{kg} / \mathrm{IV}$ saline solution, B- $250 \mu \mathrm{g} / \mathrm{kg} / \mathrm{IM}$ xylazine plus $250 \mu \mathrm{g} / \mathrm{kg} / \mathrm{IV}$ de yohimbine, C- $25 \mu \mathrm{g} / \mathrm{kg} / \mathrm{IM}$ romifidine plus $0.1 \mathrm{ml} / \mathrm{kg} / \mathrm{IV}$ saline, $D-25 \mu \mathrm{g} / \mathrm{kg} / \mathrm{IM}$ romifidine plus $250 \mu \mathrm{g} / \mathrm{kg} / \mathrm{IV}$ yohimbine. Breath rate, $\mathrm{pH}$, oxygen and carbon dioxide tensions, concentration of hydrogen carbonate, base excess and fraction of oxyhemoglobin of arterial blood were measured. A crossover experimental design was used and the comparisons of treatment means were performed by Duncan test $(P \leq 0.05)$. Xylazine and romifidine induced a decrease in partial pressure of oxygen and an increase in partial pressure of carbon dioxide in arterial blood. Yohimbine reversed the effects of xylazine and romifidine on arterial oxygen carbon partial pressures.
\end{abstract}

Keywords: goat, xylazine, romifidine, yohimbine

Recebido para publicação em 29 de agosto de 2004

Recebido para publicação, após modificações, em 10 de dezembro de 2004.

*Autor para correspondência (corresponding author).

E-mail: geraldo@vet.ufmg.br 


\section{INTRODUÇÃO}

Sintetizada em 1962, a xilazina (2- $(2,6-$ dimetilfenilamino) 5-6 dihidro-4H-1, 3-tiazina) tem sido efetiva com o propósito de viabilizar procedimentos clínicos e cirúrgicos ao produzir um conjunto de respostas comportamentais, fisiológicas e neuroquímicas, caracterizadas pela sedação, analgesia e relaxamento muscular nos animais medicados (Maze e Tranquilli, 1991). Esses efeitos se devem principalmente à ativação de receptores $\alpha_{2}$ adrenérgicos (Gasthuys et al., 1990).

A romifidina (2-[(2-bromo-6-fluorofenil) imino] monoidrocloridrato de imidazolidina) é um potente agonista $\alpha_{2}$-adrenérgico empregado na sedação de eqüinos, caninos e ovinos, apresentando maior especificidade para os adrenoceptores do tipo $\alpha_{2}$ (Gasthuys et al., 1990; Celly et al., 1997; England e Thompson, 1997). Como os agonistas $\alpha_{2}$ adrenérgicos podem causar depressão respiratória dose-dependente, a redução da freqüência respiratória, associada ao emprego da xilazina, pode ser comum (England e Clarke, 1996). Além da depressão respiratória, Doherty et al. (1986), Hsu et al. (1989) e Celly et al. (1997) verificaram a ocorrência de taquipnéia e ritmo respiratório paradoxal relacionados ao emprego da xilazina em ovinos, e Smith e Sherman (1994), em caprinos.

A redução da pressão parcial de oxigênio $\left(p a \mathrm{O}_{2}\right)$ e o aumento na pressão parcial de dióxido de carbono no sangue arterial $\left(p a \mathrm{CO}_{2}\right)$ foram avaliados após a administração de xilazina a eqüinos (Kollias-Baker et al., 1993) e caprinos (Stegmann, 1999). Os valores médios de $\mathrm{paCO}_{2}$ superior a $45 \mathrm{mmHg}$ e de $\mathrm{pH}$ menor que 7,5, após a administração de xilazina, caracterizaram a presença de hipoventilação e acidose respiratória (Haskins, 1983). O posicionamento do animal pode interferir na avaliação dos efeitos dos agonistas $\alpha_{2}$ adrenérgicos, no que se refere à $p a \mathrm{CO}_{2}$, por limitar a expansão e a perfusão pulmonares (McDonell, 1996). Segundo o autor, ovinos não sedados desenvolveram hipóxia quando colocados em decúbito lateral.

A ioimbina (17-hidroxiioimban-16-ácido carboxílico metilester), um alcalóide indólico encontrado em algumas plantas, é um antagonista dos receptores adrenérgicos do tipo $\alpha_{2}$, capaz de reverter competitivamente parte dos efeitos da xilazina (Doherty et al., 1986; Thurmon et al., 1996). A reversão dos efeitos dos agonistas $\alpha_{2}$ adrenérgicos torna-se relevante em animais de grande porte, por evitar complicações decorrentes do decúbito prolongado (Thurmon et al., 1996). Em estudos com ovinos sedados com xilazina, Doherty et al. (1986) relataram que a ioimbina melhorou a condição respiratória quanto à $p a \mathrm{O}_{2}$ e à $p a \mathrm{CO}_{2}$ e aboliu o ritmo respiratório paradoxal.

A espécie caprina é, dentre os ruminantes, a espécie mais sensível aos efeitos da xilazina (Hall e Clarke, 1987), sendo de interesse clínico a avaliação relativa ao emprego de seus antagonistas, e de outros agonistas, com maior especificidade para receptores $\alpha_{2}$ adrenérgicos. $\mathrm{O}$ objetivo deste estudo foi comparar as alterações respiratórias e hemogasométricas produzidas pela xilazina e pela romifidina, empregadas em doses equipotentes, e avaliar a eficácia da ioimbina em reverter os efeitos produzidos por esses fármacos na espécie caprina.

\section{MATERIAL E MÉTODOS}

Foram utilizadas oito cabras mestiças, hígidas, entre 2,5 e 4 anos de idade e pesos de 25 a $35 \mathrm{~kg}$, mantidas em baias individuais, alimentadas com feno de capim coast-cross (Cynodon dactylon), ração concentrada ( $16 \%$ de proteína bruta), sal mineralizado e água ad libitum. Após sete dias para adaptação ao meio ambiente experimental, os animais foram sedados com cloridrato de xilazina (Rompum $20 \mathrm{mg} / \mathrm{ml}$ - Bayer S.A.) na dose de $2,5 \mathrm{mg} / \mathrm{kg}$. Após anestesia local infiltrativa, foram submetidos à translocação cirúrgica de um segmento da artéria carótida comum esquerda para o plano subcutâneo, com o propósito de facilitar a colheita de amostras de sangue arterial. Os animais foram observados por seis semanas antes do início do experimento para assegurar a completa recuperação do trauma cirúrgico.

Foram constituídos quatro tratamentos, conforme esquema apresentado na Tab. 1. 
Tabela 1. Discriminação dos fármacos e respectivas doses administradas aos caprinos, segundo os tratamentos

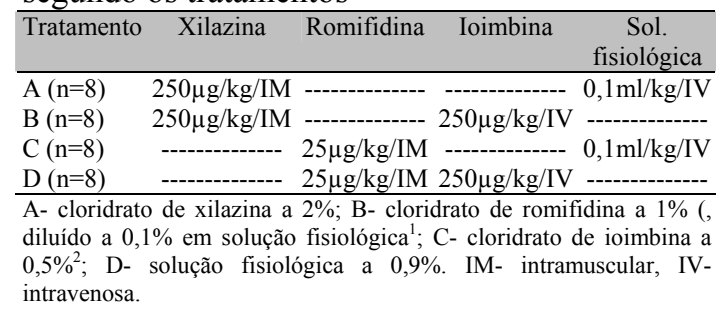

O volume de solução fisiológica foi o mesmo do aplicado para o cloridrato de ioimbina. A reversão da sedação pelo cloridrato de ioimbina foi feita 40 minutos após a aplicação dos sedativos. Todas as cabras passaram por um dos tratamentos a cada sete dias.

Foram mensuradas freqüência respiratória, $\mathrm{pH}$, pressões parciais de dióxido de carbono $\left(\mathrm{paCO}_{2}\right)$ e de oxigênio $\left(\mathrm{paO}_{2}\right)$, concentração de íon bicarbonato $\left(c \mathrm{HCO}_{3}{ }^{-}\right)$, excesso de bases ( $c$ BAse) e saturação de oxigênio $\left(s \mathrm{O}_{2}\right)$ no sangue arterial.

As cabras, submetidas a jejum alimentar e hídrico por 24 e 12 horas, respectivamente, foram contidas, colocadas em decúbito lateral direito sobre uma mesa e mantidas por cinco minutos para adaptação à posição. Em seguida, contou-se a freqüência respiratória e colheu-se sangue arterial para o estabelecimento de valores basais. Foram definidos intervalos de tempo (momentos) a partir da aplicação dos sedativos, para padronização do tempo de avaliação das variáveis (Tab. 2).

Tabela 2. Momentos padronizados para avaliação da freqüência respiratória e colheita de amostras de sangue arterial de cabras sedadas com xilazina ou romifidina e tratadas com ioimbina ou solução fisiológica

\begin{tabular}{lc}
\hline Momento & Tempo após a aplicação do sedativo \\
\hline MB & $5-15$ minutos \\
M1 & $30-40$ minutos \\
M2 & $45-55$ minutos \\
M3 & $70-80$ minutos \\
\hline
\end{tabular}

40 minutos após a aplicação do sedativo, foi administrada ioimbina ou solução fisiológica.

\footnotetext{
${ }^{1}$ Cloreto de sódio a 0,9\%- Sanobiol Ltda.

${ }^{2}$ Antagonil 5mg/ml- Wildlife Pharmaceuticals Inc.
}

A freqüência respiratória foi avaliada por inspeção dos movimentos torácicos, antes da administração dos sedativos e nos momentos 1 , 2,3 e 4 .

Para as colheitas, amostras seriadas, de sangue arterial, praticou-se uma incisão na pele com bisturi sobre a carótida translocada e introduziuse um cateter de teflon $20 \mathrm{G}^{5}$, conectado por meio de um tubo extensor com conexão rotativa ${ }^{6}$ a uma torneira de três vias ${ }^{7}$ e a uma seringa de $20 \mathrm{ml}$, contendo solução fisiológica com heparina $^{8}$, na concentração de $10 \mathrm{UI} / \mathrm{ml}$, empregada para lavagem do sistema a cada colheita de sangue.

As amostras foram colhidas de forma anaeróbica, em seringas de $3 \mathrm{ml}$ previamente heparinizadas, e acondicionadas (por no máximo duas horas) em recipiente com isolamento térmico, contendo água e gelo. As análises foram realizadas em gasômetro ${ }^{9}$ com calibração automática.

As médias dos tratamentos foram comparadas pelo teste Duncan. Os resultados foram analisados como um conjunto de dois quadrados latinos $4 \times 4$. O nível de significância para todos os resultados foi de $5 \%$.

\section{RESULTADOS}

Segundo Sampaio (2002), no planejamento estatístico crossover não é possível a comparação estatística entre os diferentes momentos de avaliação, pois os resultados de um momento são influenciados pelos dos momentos anteriores. Dessa forma, a análise estatística foi feita apenas entre os tratamentos.

Não houve diferença $(\mathrm{P}>0,05)$ entre os tratamentos (Tab. 3) com relação à freqüência respiratória, em nenhum momento de avaliação.

Os valores médios das variáveis $\mathrm{pH}, p a \mathrm{O}_{2}$ e $s \mathrm{O}_{2}$ foram menores nos tratamentos A e B em relação aos tratamentos $\mathrm{C}$ e $\mathrm{D}$ (Tab. 3), retornando a valores próximos da normalidade no tratamento

${ }^{5}$ Cateter iv 20G x 1,16in- Becton Dickinson Ind. Cir. Ltda.

${ }^{6}$ Tubo extensor com conexão rotativa de $20 \mathrm{~cm}$ - Becton Dickinson Ind. Cir. Ltda.

${ }^{7}$ Torneira de três vias- Becton Dickinson Ind. Cir. Ltda.

${ }^{8}$ Liquemine- Roche S.A.

${ }^{9}$ ABL5- Radiometer A/S. 
$\mathrm{B}$, a partir de $\mathrm{M} 3$, em resposta à administração da ioimbina. As três variáveis comportaram-se de forma semelhante ao longo do tempo, nos quatro tratamentos (Tab. 3). Houve aumento significativo da $\mathrm{paCO}_{2}$ desde $\mathrm{M} 1$ nos tratamentos $\mathrm{A}$ e $\mathrm{B}$ e redução no tratamento $\mathrm{B}$ desde M3, faixa a partir da qual pôde se avaliar o efeito da ioimbina. Não foram verificadas diferenças $(\mathrm{P}>0,05)$ entre os tratamentos quanto à $c \mathrm{HCO}_{3}^{-} \mathrm{e}$ ao $c$ Base.

Tabela 3. Valores médios da freqüência respiratória, do $\mathrm{pH}$, das pressões parciais de $\mathrm{CO}_{2}$ e $\mathrm{O}_{2}$, da concentração de íon bicarbonato, do excesso de bases e da saturação de $\mathrm{O}_{2}$ no sangue arterial de caprinos, segundo o tratamento e o momento

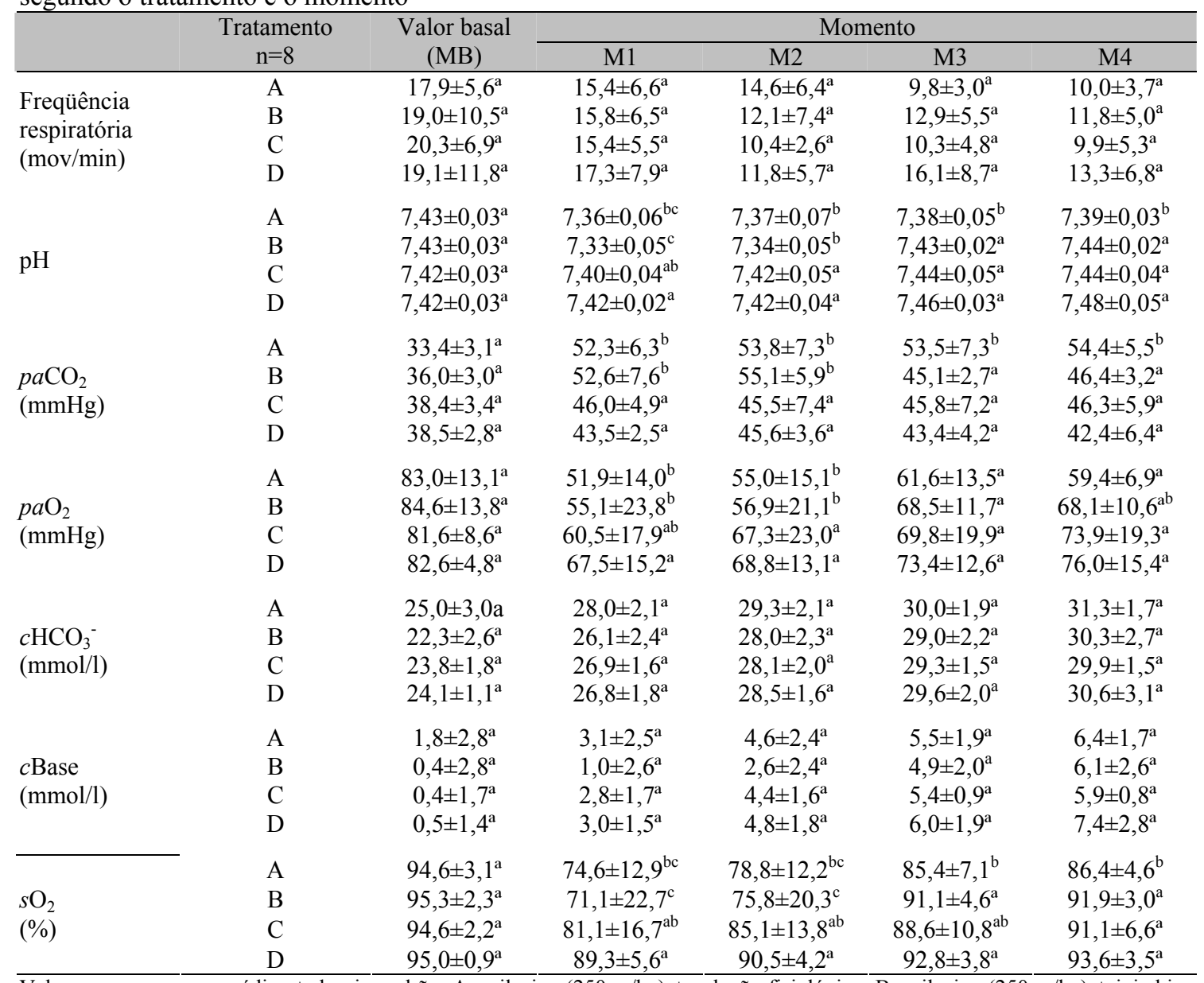

Valores expressos em médias \pm desvio-padrão. $\mathrm{A}=$ xilazina $(250 \mu \mathrm{g} / \mathrm{kg})+$ solução fisiológica; $\overline{\mathrm{B}}=$ xilazina $(250 \mu \mathrm{g} / \mathrm{kg})+$ ioimbina $(250 \mu \mathrm{g} / \mathrm{kg}): \mathrm{C}=$ romifidina $(25 \mu \mathrm{g} / \mathrm{kg})+$ solução físiológica; $\mathrm{C}=$ romifidina $(25 \mu \mathrm{g} / \mathrm{kg})+$ ioimbina $(250 \mu \mathrm{g} / \mathrm{kg})$.

Momentos estabelecidos a partir da aplicação dos sedativos: M1 $=5$ a 15 minutos; M2 $=30$ a 40 minutos; M3 $=45$ a 55 minutos; $\mathrm{M} 4=70$ a 80 minutos.

Médias com letras distintas na mesma coluna, para cada variável, diferem entre si pelo teste Duncan $(\mathrm{P} \leq 0,05)$.

\section{DISCUSSÃO}

Xilazina e romifidina não produziram alterações relevantes na freqüência respiratória. Nos gases sangüíneos as alterações de maior magnitude foram relacionadas ao emprego da xilazina, isto é, observou-se aumento da $p a \mathrm{CO}_{2}$ e diminuição da $p a \mathrm{O}_{2}$ e $\mathrm{sO}_{2}$ entre os momentos 1 e 2. Os valores médios de $p \mathrm{CO}_{2}$ acima de $45 \mathrm{mmHg}$ e de $\mathrm{pH}$ abaixo de 7,5, após a administração de xilazina, caracterizaram a ocorrência de hipoventilação e acidose respiratória, como observado por Haskins (1983). Uma justificativa possível para essa ocorrência é o fato de a 
xilazina aumentar a resistência das vias aéreas (McDonell, 1996). Aumento na $p a \mathrm{CO}_{2}$ também pôde ser observado após a administração de romifidina, quando os valores foram comparados aos basais, embora os valores tenham permanecido próximos ao limite superior da variação considerada fisiológica (35 a $45 \mathrm{mmHg}$ ), segundo Muir et al. (1995).

Redução dos valores de $p a \mathrm{O}_{2}$ foi verificada nos quatro tratamentos, mas a exemplo da $p a \mathrm{CO}_{2}$, as oscilações foram mais acentuadas nos tratamentos com xilazina. Nestes, os valores de $p a \mathrm{O}_{2}$ foram inferiores a $60 \mathrm{mmHg}$, o que pode caracterizar hipoxemia, assemelhando-se às observações feitas em ovinos sedados com xilazina por Doherty et al. (1986) e Hsu et al. (1989). Podem ser causas dessa alteração a hipoventilação, atribuída ao aumento verificado na $p a \mathrm{CO}_{2}$, o estabelecimento de shunts artériovenosos na vasculatura pulmonar e a existência de áreas alveolares colabadas ou hipofuncionais (Haskins, 1983). Independente da causa, a redução dos valores de $p a \mathrm{O}_{2}$, observada nos quatro tratamentos, parece estar associada ao efeito agonista dos medicamentos utilizados sobre os receptores adrenérgicos, já que houve uma resposta positiva após a tentativa de reversão pelo emprego da ioimbina.

As diferenças entre o emprego de xilazina e romifidina puderam ser observadas quanto ao $\mathrm{pH}$, à $p a \mathrm{CO}_{2}$, à $p a \mathrm{O}_{2}$ e à $s \mathrm{O}_{2}$ no sangue, o que difere das observações feitas em ovinos por Celly et al. (1997). Embora esses fármacos sejam diferentes quanto à especificidade por receptores $\alpha_{2}$ - adrenérgico (England e Clarke, 1996), eles foram empregados em doses de potência aproximada, portanto, as diferenças podem estar relacionadas ao efeito agonista mais intenso da xilazina em receptores $\alpha_{1}$ - adrenérgico ou a critérios na seleção de doses equipotentes (Massone et al., 1993). O decúbito dos animais foi considerado agravante em diversas pesquisas (McDonell, 1996), mas neste estudo o posicionamento não interferiu na comparação entre os tratamentos.

$c \mathrm{HCO}_{3}^{-} \mathrm{e} c$ Base, cujos valores normais são $24 \pm 3 \mathrm{mmol} / 1$ e $0 \mathrm{mmol} / \mathrm{l}$, respectivamente (Haskins, 1983), são componentes metabólicos do equilíbrio ácido-base. Neste estudo não houve diferença entre os tratamentos, e o aumento de ambas as variáveis ao longo do tempo de avaliação foi uma resposta metabólica compensatória às alterações do $\mathrm{pH}$ e da $\mathrm{paCO}_{2}$.

\section{CONCLUSÕES}

Nas condições deste experimento, pode-se concluir que: a xilazina produz alterações mais significativas no $\mathrm{pH}$, na $p a \mathrm{CO}_{2}$ e na $p a \mathrm{O}_{2}$, do que a romifidina; a ioimbina reverte as alterações na pressão parcial de $\mathrm{O}_{2}$ e $\mathrm{CO}_{2}$ produzidas pela xilazina.

\section{AGRADECIMENTOS}

A Becton Dickinson Indústrias Cirúrgicas Ltda e a Wildlife Pharmaceiticals Incorporated pela doação de parte do material utilizado neste estudo.

\section{REFERÊNCIAS BIBLIOGRÁFICAS}

CELLY, C.S.; McDONELL, W.N.; YOUNGS, S.S. et al. The comparative hypoxemic effect of four $\alpha_{2-}$ adrenoceptor agonists (xylazine, romifidina, detomidine and medetomidine) in sheep. J. Vet. Pharmacol. Therap., v.20, p.464$471,1997$.

DOHERTY, T.J.; PASCOE, P.J.; McDONELL, W.N. et al. Cardiopulmonary effects of xylazine and yohimbine in laterally recumbent seep. Can. J. Vet. Res., v.50, p.517-521, 1986.

ENGLAND, G.C.W.; THOMPSON, S. The influence of route of administration upon the sedative effect of romofidine in dogs. J. Vet. Anaesth., v.24, p.21-24, 1997.

ENGLAND, G.C.W.; CLARKE, K.W. Alpha adrenoceptor agonists in the horse- a review. $\mathrm{Br}$. Vet. J., v.152, p.641-657, 1996.

GASTHUYS, F.; PARMENTIER, D.; GOOSSENS, L. et al. A preliminary study on the effects of atropine sulphate on bradycardia and heart blocks during romifidine sedation in horse. Vet. Res. Commun., v.14, p.489-502, 1990.

HAL, L.W.; CLARKE, K.W. Anestesia veterinária. 8.ed. São Paulo: Manole, 1987, p.5176: Principles of sedation, analgesia and premedication. 
HASKINS, S.C. Blood gases and acid- base balance: clinical interpretation and therapeutical implications. In: KIRK, R. W. (Ed.). Current veterinary therapy. v.8. Philadelphia: W.B. Saunders, 1983. v.8, p.201-215.

HSU, W.H.; HANSON, C.E.; HEMBROUGH, F.B. et al. Effects of iadoxan, tolazoline, and yohimbine on xylazine- induced respiratory changes and central nervous system depression in ewes. Am J. Vet. Res., v.50, p.1570-1573, 1989.

KOLLIAS- BAKER, C.A.; COURT, M.H.; WILLIAMS, L.L. Influence of yohimbine and tolazoline on the cardiovascular, respiratory and sedative effects of xylazine in the horse. J. Vet. Pharmacol. Therap., v.16, p.350-358, 1993.

MASSONE, F.; LUNA, S.P.; CASTRO, G.B. et al. Sedation with romifidine or xylazine in cattle, is the same? Anaesthesiology, v.20, p.55, 1993.

MAZE, M.; TRANQUILI, W. Alpha adrenoceptor agonists: defining the role in clinical anesthesia. Anesthesiology, v.74, p.581$605,1991$.

McDONELL, W. Respiratory system. In: THURMON, J.C.; TRANQUILI; W.J.,
BENSON, G.J. Lumb e Jone's veterinary anesthesia. 3.ed. Urbana: Williams e Wilkins, 1996. p.115-147.

MUIR, W.W.; HUBBELL, J.A.E.; SKARDA, R.T. et al. Handbook of veterinary anesthesia. 2.ed. St. Louis:Mosby, 1995. p.227-261: Patient monitoring during anesthesia.

SAMPAIO, I.B.M.; Estatística aplicada à experimentação animal. 2.ed. Belo Horizonte: FEPMVZ, 2002. 265p.

SMITH, M.C.; SHERMAN, D.M. Goat medicine. Philadelphia: Lea e Febiger, 1994. p.509-517: Anesthesia.

STEGMANN, G.F. Observations on some cardiopulmonary effects of midazolam- xylazine and midazolan- ketamine combination in the goat. J. S. Afr. Vet. Assoc., v.70, p.122-126, 1999.

THURMON, J.C.; TRANQUILLI, W.J.; BENSON, G.J. Lumb e Jone's veterinary anesthesia. 3.ed. Urbana: Williams e Wilkins, 1996. p.183-209: Preanesthetics and anesthetic adjuncts. 